\title{
Effect of Cylindrical Turbulators on the Mixing Process in Basic Venturi Gas Mixer Using OpenFOAM
}

\author{
Mathias Romańczyk ${ }^{1, *}$, and Witold Elsner ${ }^{1}$ \\ ${ }^{1}$ Czestochowa University of Technology, Institute of Thermal Machinery, al. Armii Krajowej 21, 42-201 Czestochowa, Poland
}

\begin{abstract}
This study was devoted to improving the mixing quality in a Venturi gas mixer. It is well known that the performance of industrial gas engines strongly depends on the quality of air and fuel mixture and therefore on its homogeneity. Incorrect mixture proportions may lead to unstable operation of the entire industrial gas engine and excessive emissions exceeding environmental standards of the European Union. The effectiveness of the mixing process in a Venturi gas mixer depends on many factors, i.e. correct proportions between Air inlet, Venturi Throat diameter, as well as gas inlet area. To improve the intensification of the whole mixing process, as well as to increase the mixing time between gas and air, it was decided to insert disturbing elements in form of cylindrical turbulators into the Venturi pipe. Therefore numerical calculations were performed for the most commonly consumed by industrial gas engines lean Air-Fuel mixture, with an air excess coefficient ratio of $\lambda=$ 1.6. In numerical analysis, OpenFOAM software was used to investigate the impact of cylindrical turbulators on the whole mixing process in a basic Venturi gas mixer. Particular attention was focused on the Air-Fuel Ratio (AFR) changes, Turbulent Kinetic Energy (TKE) and Pressure loss [Pa], which is one of the most qualitative evaluation parameters of a gas mixer.
\end{abstract}

\section{Introduction}

Environmental standards of the European Union have been increasingly tightened over the last years, which puts the energy industry ahead of a big challenge. In this sector, industrial gas engines perform very well when it comes to renewable energy sources (such as Biogas) giving off relatively low exhaust gas emissions, which depends on the proper operation of such an engine. The performance of industrial gas engines is widely known to strongly depend on the quality of mixing air and fuel, and therefore the homogeneity of the mixture. Rapid variations of concentrations between air and fuel called "Air-Fuel Ratio (AFR) changes" have a huge negative impact not only on environmental emissions but also on fuel consumption in industrial gas engines [1]. Therefore a gas mixer should prepare a mixture of appropriate air/fuel concentration, depending on the demand of a given gas engine - from no load during ignition time to full load conditions when working under maximum performance [2]. Improper mixture proportion can lead to unstable operation of the gas engine, as well as excessive environmental emissions. It is known, that for industrial gas engines, the anomalies related to combustion processes significantly differ from those of conventional combustion processes. These processes may negatively affect the operation of the industrial gas engine, which is associated with many types of undesirable phenomena [3]. How the entire combustion process develops has a huge impact on the appropriate proportion of air-fuel mixture [4]. To ensure an efficient combustion process in an industrial gas engine, the design of the Venturi mixer should ensure the best possible mixing of the two components. In addition, it should be compact, with minimal pressure loss and in good suction pressure in the throat, due to the Venturi principle. Over the last decade, different analyses have been undertaken to improve the efficiency of the entire mixing process in a Venturi gas mixer. These analyses were devoted to its different geometrical parameters such as the Venturi throat diameter investigated by Danardono et al. [5] or the gas inlet area, where the optimal gas inlet localisation [6] and the influence of gas inlet angle on mixing behaviour in a Venturi gas mixer [7] were investigated. For further improvement of the mixing efficiency in a Venturi gas mixer it was decided to place a cylindrical turbulator (a device, which increases local the turbulence of the fluid) $\varnothing 20$ [mm] transverse to the flow axis of the Venturi gas mixer to examine the influence of the turbulator on the entire mixing process. The expected effect of such an operation was to intensify the mixing process. Therefore in this paper was investigated the influence of turbulators on the mixing process in a basic Venturi gas mixer using the open source computational fluid dynamics simulation software OpenFOAM.

\section{Venturi mixer design and OpenFOAM numerical modelling software}

More and more manufacturers of gas engine equipment are attempting to design gas mixers based on the Venturi

\footnotetext{
Corresponding author: mathias@imc.pcz.czest.pl
} 
principle. The Venturi principle is described by the drop in fluid pressure occurring when a fluid flows through a constricted section (Throat) in this pipe. In fluid dynamics, fluid velocity $\left(U_{x}\right)$ increases as it passes through a throat - in accordance with the principle of mass continuity, while its static pressure $\left(p_{s}\right)$ decreases - in accordance with the principle of the conservation of mechanical energy. The decrease at a certain point (Venturi Throat) in the cross-sectional area of this pipe (see Fig. 1), in accordance with Bernoulli's law and the continuity equation, creates vacuum resulting in gas being sucked into the Venturi gas mixer through the gas inlet. In this way gas is being mixed with air.

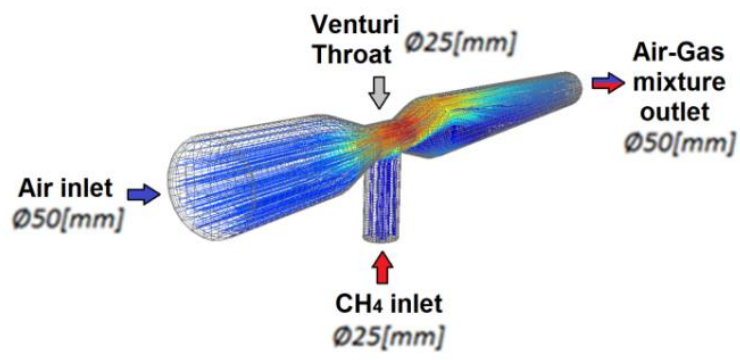

Fig. 1. Venturi gas mixer design with dimensions [mm].

The effectiveness of the mixing process in a Venturi gas mixer depends on many interrelated geometrical parameters, such as the Venturi Throat in connection with the gas inlet area and its position. To improve the intensification of the mixing, as well as to increase the mixing time between gas and air, it was decided to change the geometry of the Venturi gas mixer by implementing some disturbing elements in the form of Ø20 [mm] cylindrical turbulators (see Fig. 2) in the pipe after the gas mixer throat. The objective of this modification was to intensify the mixing process with simultaneous increasing the mixing time of gas and air. To this end, three gas mixer designs were numerically compared: a standard Venturi gas mixer (ST), Venturi gas mixer with one turbulator (TP1) and with two turbulators (TP1 and TP2) at a distance of $0.1[\mathrm{~m}]$ from each other.

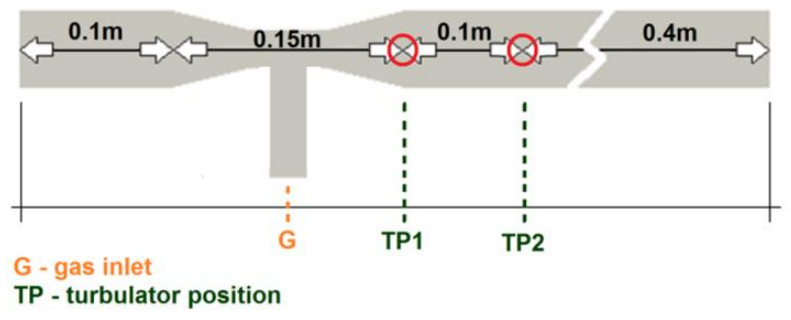

Fig. 2. Analysed cases with marked positions of gas inlet (G), turbulator positions (TP1, TP2) and dimensions [mm].

The Venturi gas mixer models were designed with the software Autodesk Inventor. The dimensions were adopted in all three analysed cases at the same level (see Fig. 1 and Fig. 2). Differences resulted only from the arrangement of the turbulators $(100[\mathrm{~mm}]$ from each other) in the two modified mixers. The air inlet was 50 $[\mathrm{mm}]$ in diameter and $100[\mathrm{~mm}]$ in length. The Venturi Throat point, as well as the gas inlet $\left(\mathrm{CH}_{4}\right)$, had a diameter of $\rightarrow 25[\mathrm{~mm}]$. The point of the Throat was centred on the next $150[\mathrm{~mm}]$ past the air inlet, while the air/gas mixing tube after passing the Venturi Throat was specified to take $500[\mathrm{~mm}]$ in length to achieve adequate convergence of the entire numerical simulation. For this reason, the total length of the analysed Venturi gas mixers was defined to be 750 [mm]. Numerical calculations were performed for a lean air/gas combustion mixture, in which the most industrial gas engines operate, and where the air excess coefficient of it amounts to $\lambda=1.6$. In the numerical analysis, the OpenFOAM software was used. It is well known, that the flow through a Venturi gas mixer is turbulent, so it was necessary to implement in this numerical calculations the turbulence model. For this purpose, a two-equation turbulence model $\mathrm{k}-\varepsilon$ was used to analyse the distribution of Turbulence Kinetic Energy (TKE) along the Venturi gas mixer. The turbulence model was selected because it is one of the most widely used and well-validated models in terms of consistency and reliability [8]. To analyse the efficiency of the air/gas mixing process, a modified OpenFOAM solver reactingFoam was used. This model is based on the Volume of Fluid (VOF) methodology, which allows analysing different mixing reactions. In this research, the mixing of methane $\rightarrow \mathrm{CH}_{4}$ and air was analysed.

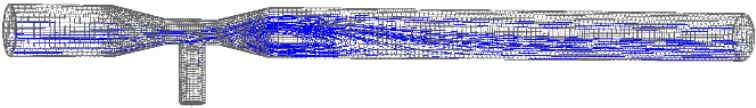

b)

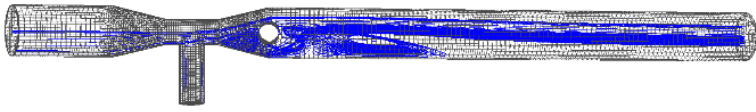

c)

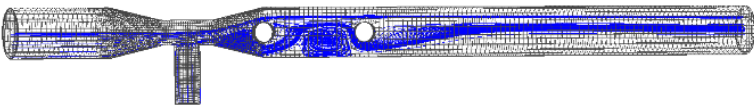

Fig. 3. Streamline distribution - a) ST - without turbulator (ST - Standard Venturi mixer), b) T1 - with one turbulator 20 [mm], c) $\mathrm{T} 2$ - with two turbulators $2 \times 20$ [mm].

Analysing the streamline distributions in Fig. 3 one may notice the disturbing effect caused by the use of such a turbulator. The turbulator causes the movement of the vortex concentration in a further distance. The use of two turbulators behind each other causes a strongly visible vortex generation before the second turbulator. The goal of such an operation was to intensify the entire mixing process, as well as to increase the air/gas mixing time. The detailed analysis showed that the intensification between mixing air and gas increases rapidly. The mixing process was extended, while on the end of the Venturi gas mixer the trace of the mixture appeared considerably heterogeneous.

\section{Results and discussion}

The results presented in this paper show a comparison between three mixer designs, one standard Venturi gas mixer, and two mixer designs equipped with cylindrical turbulators, the disturbing elements, at a distance of 0.1 [m] from each other, as shown in Fig. 2. Particular attention was paid to the Air-Fuel Ratio changes (AFR), based on analysis of methane mass fraction $\rightarrow \mathrm{CH}_{4}$ concentration distribution through the Venturi gas mixer 
in average numerical data and cross-sectional views. Furthermore, due to the turbulator's impact on flow conditions, it was resolved that further analyses should include the distribution of Turbulence Kinetic Energy (TKE) throughout the Venturi gas mixer, as well as pressure loss $[\mathrm{Pa}]$, which is one of the most qualitative parameters to describe the quality and efficiency of a gas mixer. This section will report on the influence of turbulator(s) on the mixing process in a basic Venturi gas mixer using the software OpenFOAM. For the sake of validation of this numerical data - mathematical calculations were carried out according to the literature data of the Air-Fuel Ratio for a lean air/gas combustion mixture, typical for industrial gas engines, with an air excess coefficient ratio of $\rightarrow \lambda=1.6$, where the stoichiometric (i.e. $\lambda=1.0$ ) Air-Fuel Ratio $A F R_{\text {stoich }}$ for methane $\mathrm{CH}_{4}$ equals $9.52 \frac{m_{\text {air }}}{m_{\text {fuel }}}$ [9]. The mathematical calculation is given below:

$$
\begin{aligned}
A F R & =\frac{m_{\text {air }}}{m_{\text {fuel }}} \\
A F R_{\text {stoich }} & =9.52 \frac{m_{\text {air }}}{m_{\text {fuel }}}
\end{aligned}
$$

where the Air excess coefficient ratio $\rightarrow \lambda$ :

$$
\lambda=\frac{A F R}{A F R_{\text {stoich }}}
$$

and for $\rightarrow \lambda=1.6$ :

and as a result:

$$
1.6=\frac{A F R}{9.52} \Rightarrow A F R=15.232 \frac{m_{\text {air }}}{m_{\text {fuel }}}
$$

$$
\text { for } A F R=15.232 \frac{m_{\text {air }}}{m_{\text {fuel }}} \Rightarrow \frac{0.9384 m_{\text {air }}[-]}{0.0616 m_{C_{4}}[-]}
$$

The performed mathematical calculations show that: for an Air excess coefficient ratio of $\lambda=1.6$, in which the most industrial gas engines operate, the concentration of methane $\rightarrow \mathrm{CH}_{4}$ constitutes about 6 [\%] of the whole mixture: $\mathrm{CH}_{4} \Rightarrow 0.0616 \mathrm{~m}_{\mathrm{CH}_{4}}$ while air approx. the remaining 94 [\%] of the whole mixture, air $\Rightarrow$ $0.9384 m_{\text {air }}$. In the next step, detailed analyses between mathematical and average numerical data were made, to show whether numerical results match the mathematical calculations.

Table 1. Comparative analysis of results from mathematical and numerical calculations with the specified relative error at the outlet of the Venturi gas mixer.

\begin{tabular}{|c|c|c|c|c|c|}
\cline { 2 - 6 } \multicolumn{1}{c|}{} & $\begin{array}{c}\text { Mix } \\
\text { type }\end{array}$ & $\begin{array}{c}\text { Concentration } \\
\text { of air }\end{array}$ & $\begin{array}{c}\text { Concentration } \\
\text { of } \mathrm{CH}_{4}\end{array}$ & $\begin{array}{c}\text { Air excess } \\
\text { coefficient } \\
\rightarrow \lambda\end{array}$ & $\begin{array}{c}\text { Relative } \\
\text { error }\end{array}$ \\
\hline Units & {$[-]$} & {$[-]$} & {$[-]$} & {$[-]$} & {$[\%]$} \\
\hline $\begin{array}{c}\text { Math. } \\
\text { calculations }\end{array}$ & $\mathrm{ST}$ & 0.93840 & 0.06160 & 1.60000 & 0.00 \\
\hline \multirow{2}{*}{$\begin{array}{c}\text { Numerical } \\
\text { calculations }\end{array}$} & $\mathrm{ST}$ & 0.93835 & 0.06165 & 1.59888 & 0.07 \\
\cline { 2 - 6 } & $\mathrm{T} 1$ & 0.93968 & 0.06032 & 1.63695 & 2.31 \\
\cline { 2 - 6 } & $\mathrm{T} 2$ & 0.94079 & 0.05921 & 1.66888 & 4.30 \\
\hline
\end{tabular}

In addition, the relative error was calculated for the outlet of the Venturi gas mixer to show the deviation between the results of numerical simulation and mathematical calculations, where ST - is the standard Venturi gas mixer, T1 - is the gas mixer equipped with one turbulator $\varnothing 20[\mathrm{~mm}]$ located immediately after the Venturi Throat of the gas mixer and T2 - is the gas mixer equipped with two turbulators Ø20 [mm], one immediately after the Venturi Throat of the gas mixer and the other $0.1[\mathrm{~m}]$ further into the mixing pipe. The relative error was calculated using the formula:

$$
\delta=\frac{\Delta x}{x}=\frac{\left|x-x_{o}\right|}{x}
$$

Often the relative error is expressed as a percentage, which is when we the following formula applied:

$$
\delta=\frac{\Delta x}{x} \cdot 100 \%=\frac{\left|x-x_{o}\right|}{x} \cdot 100 \%
$$

where:

$\Delta x$ - is the absolute error of the obtained result, $x$ - is the exact value to which we compare, $x_{o}-$ is the value we compare.

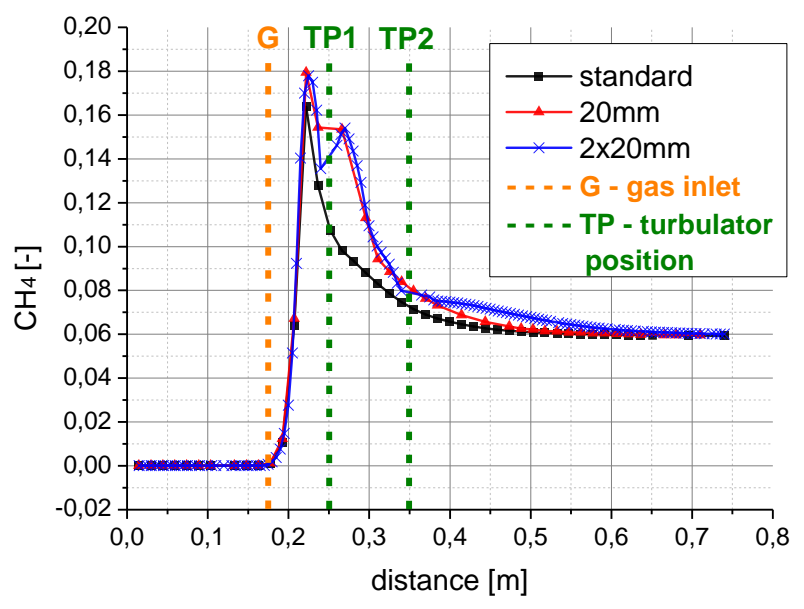

Fig. 4. Concentration of methane mass fraction $\mathrm{CH}_{4}$ through the Venturi gas mixer for three analysed cases.

The data in Table 1, describing the concentrations of air and methane mass fraction $\rightarrow \mathrm{CH}_{4}$ through the Venturi gas mixer, prove that there is a full compatibility of the results calculated with theoretical mathematical formulas with the numerical calculations performed for the standard Venturi gas mixer using OpenFOAM software. The approximation error, in this case, amounts to mere 0.07 [\%], and therefore it can be concluded that the numerical calculations were performed correctly for these three analysed cases. It can be seen that the relative error is greater for the gas mixers equipped with turbulators. The biggest relative error of 4.30 [\%] is for the gas mixer equipped with two turbulators. This may indicate a strong flow disturbance, which is associated with the effect of a strong aerodynamic trace, which arises through strong turbulence. The strong disturbance causes a little less methane $\rightarrow \mathrm{CH}_{4}$ sucked into the Venturi gas mixer with a delay which causes a poorer mixture proportion at the outlet of the Venturi mixer as evidenced by a greater Air excess coefficient ratio up to $\lambda \approx 1.67$. Fig. 4 shows 
average concentrations of methane mass fraction changing through the Venturi gas mixers for different analysed cases. Analysing Fig. 4 it can be noted that the intensification between mixing air and gas increases rapidly after the first turbulator (TP1) in both cases of Venturi gas mixers that are equipped with turbulators. Analysing the whole mixing process in a Venturi gas mixer, it is of high importance to know, how the concentrations of methane mass fraction $\rightarrow \mathrm{CH}_{4}$ and air change through the whole mixer section. Therefore mixing characteristics in a cross-sectional view of the Venturi gas mixer were analysed in Fig. 5 after two turbulators at a distance of 0.45 [m], and in Fig. 6 at the outlet of the Venturi gas mixer.

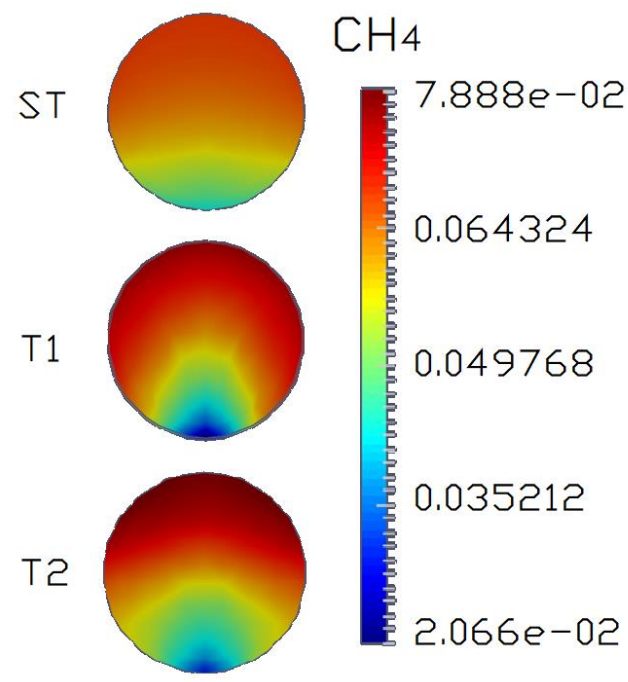

Fig. 5. Distribution of methane mass fraction $\rightarrow \mathrm{CH}_{4}[-]$ in crosssectional view after two turbulators at distance $0.45[\mathrm{~m}]$ from the inlet -ST) without turbulator, T1) with one turbulator 20 [mm], T2) with two turbulators $2 \times 20[\mathrm{~mm}]$.

Analysed in Fig. 5 distributions of methane mass fraction $\mathrm{CH}_{4}$ [-] in cross-sectional view after two turbulators at distance $0.45[\mathrm{~m}]$ from the inlet for this three analysed cases exhibit higher disturbances spectrum in the cases of $\mathrm{T} 1$ and $\mathrm{T} 2$, while in the standard Venturi gas mixer ST occurs a smooth distribution. Analysing Figs. 4 and 5 it can be noticed, that the whole mixing process has been extended, while at the end of the Venturi gas mixer the trace of the mixture looks very heterogeneous in the T1 and T2 (see Fig. 6). The most heterogeneous mixture is obtained in T2 after two turbulators, where the flow disturbances are the greatest.

As it was mentioned before, the flow through a Venturi gas mixer is turbulent. In addition, introducing additional disturbing elements to the Venturi gas mixer in the form of turbulators increases strongly the intensification of the mixing process. Therefore Turbulent Kinetic Energy (TKE) distribution along the flow through the Venturi gas mixer for this three analysed cases requires close consideration. Fig. 7 shows the distribution of Turbulent Kinetic Energy (TKE). Analysis of Fig. 7 reveals that there is a drastic drop of the turbulent fluctuations (TKE) after passing the Venturi Throat of the gas mixer. The biggest drop occurs in the Venturi gas mixer equipped with two turbulators.

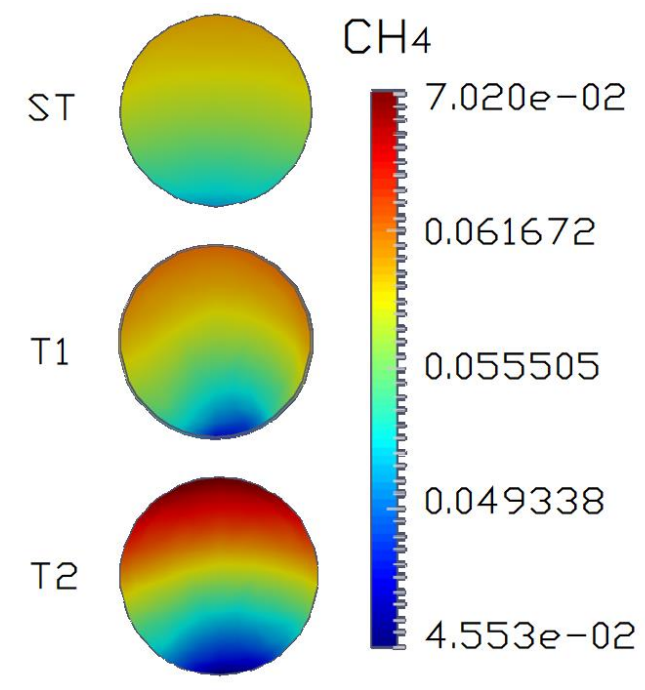

Fig. 6. Distribution of methane mass fraction $\rightarrow \mathrm{CH}_{4}[-]$ in crosssectional view at the outlet of the Venturi gas mixer at distance $0.74[\mathrm{~m}]$ from the inlet. $-\mathrm{ST}$ ) without turbulator, T1) with one turbulator $20[\mathrm{~mm}], \mathrm{T} 2)$ with two turbulators $2 \times 20[\mathrm{~mm}]$.

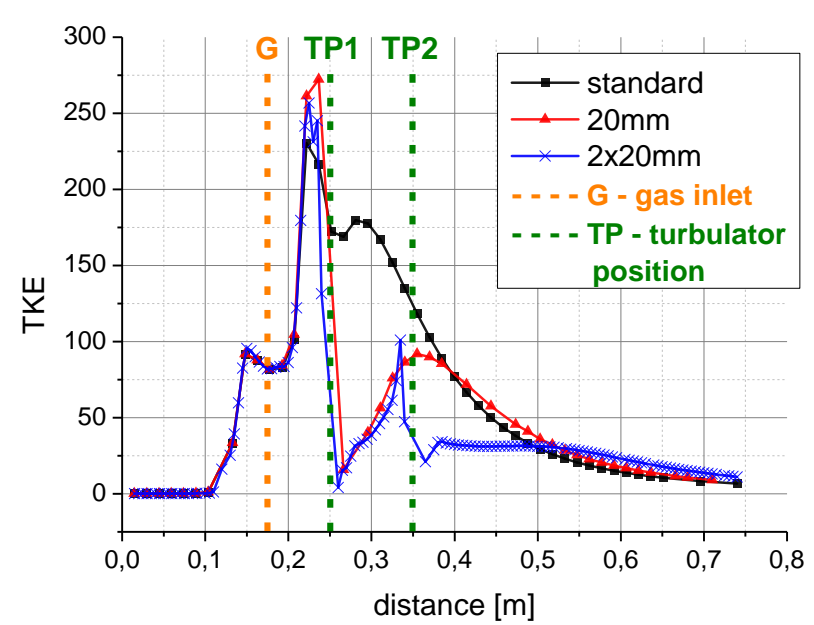

Fig. 7. Distribution of Turbulent Kinetic Energy (TKE) through the Venturi gas mixer for three analysed cases.

In fluid dynamics, Turbulent Kinetic Energy (TKE) is defined as the mean kinetic energy per unit mass associated with eddies in a turbulent flow. Physically, TKE is characterised by Root-Mean-Square (RMS), in other words - velocity fluctuations. When the flow rate increases, there occurs a drastic growth in turbulent fluctuations. The biggest whirl affects and draws energy from the main flow. The forces of inertia dominate, while the viscosity forces are negligible. In numerical calculations, turbulence kinetic energy (TKE) can be calculated based on the closure method, i.e. a turbulence model. These models are based on the ReynoldsAveraged Navier Stokes equations (RANS). As it was mentioned before, in numerical calculations the turbulence model $\mathrm{k}-\varepsilon$ was applied. Turbulence Kinetic Energy (TKE) can be generated by fluid shear, friction, buoyancy, or through an external force at low-frequency eddy scales [10]. 


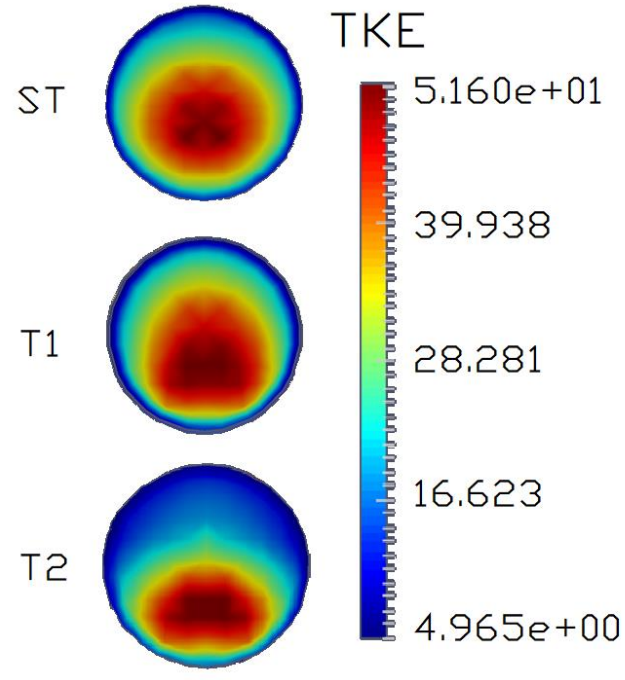

Fig. 8. Distribution of Turbulent Kinetic Energy $\rightarrow$ TKE in cross-sectional view after two turbulators at distance $0.45[\mathrm{~m}]$ from the inlet. $-\mathrm{ST}$ ) without turbulator, T1) with one turbulator 20 [mm], T2) with two turbulators $2 \times 20$ [mm].

Fig. 8, showing the distribution of Turbulent Kinetic Energy $\rightarrow$ TKE in cross-sectional view after two turbulators at distance $0.45[\mathrm{~m}]$ from the inlet for all three analysed cases, indicates that the energy trace is strongly shifted to the wall in the Venturi gas mixer equipped with two turbulators - T2, which indicates a strong destabilisation of the flow, as noted in Fig. 5 - the crosssectional distribution of methane mass fraction; perhaps the processes are strongly related to each other. Finally, the most significant parameter of the Venturi gas mixer the pressure loss was analysed. This is one of the most qualitative parameters to describe the quality and efficiency of a gas mixer. Firstly, the average numerical data of the pressure loss through the Venturi mixer for all analysed cases is shown in Fig. 9. Considering the pressure loss $[\mathrm{Pa}]$ through the Venturi gas mixer for all three analysed cases, it could be noted that the largest pressure loss through the whole Venturi mixer occurs in the gas mixer equipped with two turbulators.

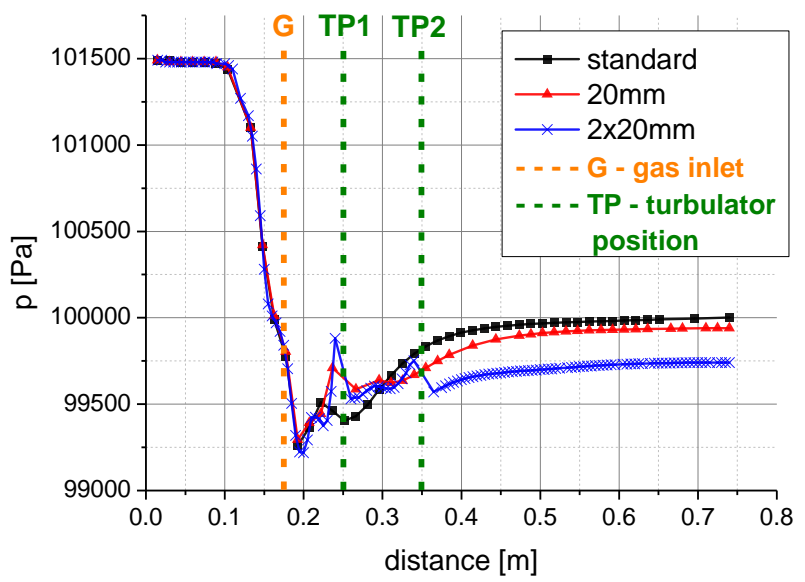

Fig, 9. Pressure loss [Pa] through the Venturi gas mixer for three analysed cases.

It could be also noted, that the entire pressure loss occurs in the Venturi throat of the gas mixer, after which it begins to grow slowly again until the distance of $0.6[\mathrm{~m}]$ from the Venturi mixer inlet where it stabilises and maintains a constant level for each analysed case.

It should moreover be noted that the most violent pressure change occurred before the first cylindrical turbulator. This huge pressure difference seen in Fig. 9 is mainly caused by the detachment of the flow streams from the cylindrical turbulators, which causes a strong turbulence with the formation of vortices.

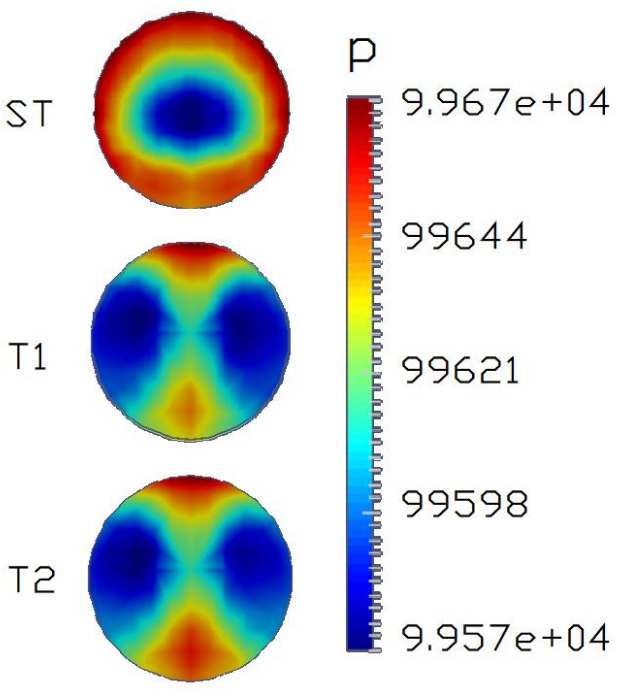

Fig. 10. Distribution of pressure loss $\rightarrow p[\mathrm{~Pa}]$ in cross-sectional view after one turbulator at distance $0.3[\mathrm{~m}]$ from the inlet. $-\mathrm{ST}$ ) without turbulator, T1) with one turbulator $20[\mathrm{~mm}], \mathrm{T} 2)$ with two turbulators $2 \times 20[\mathrm{~mm}]$.

Fig. 10 shows the effect caused by the flow around a circular cylinder, exhibiting distinct over- and subpressure regions (T1 and $\mathrm{T} 2$ ). This huge pressure difference is mainly attributed to the detachment of the flow streams from the cylinder (cylindrical turbulator) and the formation of vortices. All the energy of the flow stream turns into potential energy of static pressure. The pressure assumes the maximum value. Points in which the velocity assumes zero values $\left(U_{x}=0\right)$, i.e. the points of accumulation. In the point of accumulation the stream splits into two turbulating branches flowing around the cylinder. This process causes a strong destabilisation of the entire mixing process. Fig. 11 presents the distribution of pressure loss $\rightarrow \mathrm{p}[\mathrm{Pa}]$ in cross-sectional view after two turbulators at distance $0.45[\mathrm{~m}]$ from the inlet and what becomes evident is the exceptionally chaotic pressure distribution.

In addition, this chaotic pressure distribution indicates a strong flow disturbance, which is associated with the effect of strong aerodynamic traces caused by the cylindrical turbulators. This arises mainly through a strong turbulence in this flow. Such a flow destabilisation process is strongly affected by the relationship between the Turbulent Kinetic Energy (TKE) and pressure loss. It also affects the Air-Fuel Ratio changes (AFR), which could be seen in the analysis of the methane mass fraction distribution in the cross-sectional view in Fig. 5 and Fig. 6. 


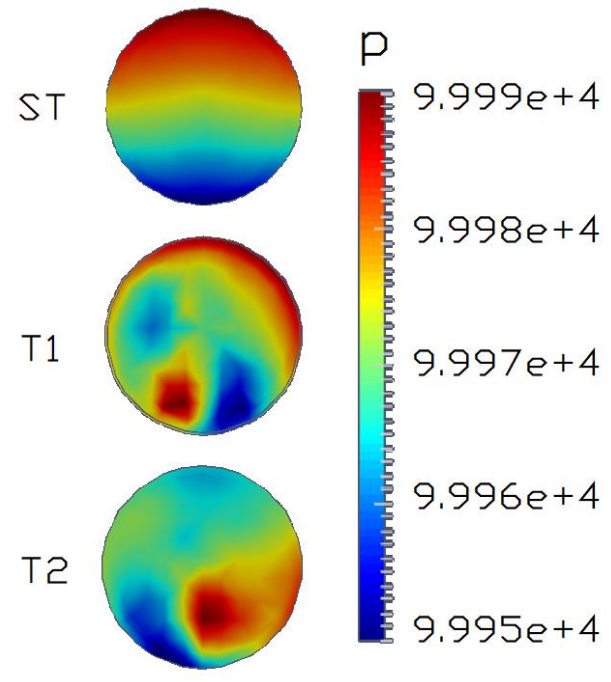

Fig. 11. Distribution of pressure loss $\rightarrow p$ [Pa] in cross-sectional view after two turbulators at distance $0.45[\mathrm{~m}]$ from the inlet. ST) without turbulator, T1) with one turbulator 20 [mm], T2) with two turbulators $2 \times 20$ [mm].

\section{Conclusions}

This paper investigated the influence of cylindrical turbulators on the entire mixing process in a basic Venturi gas mixer, using the open source CFD software OpenFOAM. The objective of this modification was to boost intensification of the whole mixing process, as well as to increase the air/gas mixing time. For the sake of validation, mathematical calculations were performed according to the literature data on the Air-Fuel Ratio for a typical lean air/gas combustion mixture for industrial engines with air excess coefficient ratio of $\rightarrow \lambda=1.6$. The results calculated with theoretical mathematical formulas show full compatibility with the average numerical calculations performed using the CFD simulation software OpenFOAM. This study has shown that the intensification of air/gas mixing has increased rapidly. The mixing process was extended, while at the end of the Venturi gas mixer, the mixing trace exhibited considerable heterogeneity (see Fig. 6). The detailed analysis presented in this paper showed that the proposed turbulator introduces strong flow disturbances resulting in strong aerodynamic traces which in turn produce a highly heterogeneous mixture. This process also significantly affects the decrease in the efficiency of the whole mixing process. Moreover, when the Venturi gas mixer is equipped with a turbulator, a greater pressure drop occurs through the whole mixer section which is an undesirable phenomenon.

This study was supported by the Czestochowa University Grant BS/MN 1-103-302/2018/P.

\section{References}

1. M. Gorjibandpy, M.K. Sangsereki, World Academy of Science, Engineering and Technology. International Journal of Mechanical, Aerospace, Industrial, Mechatronic and Manufacturing Engineering, 4 (2010)

2. R. Devarajan, Comparative Evaluation of a Two Stroke Compressed Natural Gas Mixer Design using Simulation and Experimental Techniques. International Conference on Association of Science and Technology for Development, Power and Energy Systems, Langkawi, 359 (2008)

3. K.M. Han, Lichtleiterbasierte Methoden zur optischen Analyse von räumlichen Verbrennungsprozessen und Verbrennungsanomalien in Ottomotoren. Technische Universität Karlsruhe, Dissertation (2010)

4. M. Günther, Y. Uygun, F. Kremer, S. Pischinger, $M T Z$ Worldw, 74, 46 (2013)

5. D. Danardono, K.S. Kim, S.Y. Lee, J.H. Lee, J. Mech. Sci. Technol. 25 (9), 2285 (2011)

6. M. Romańczyk, W. Elsner, $23^{\text {rd }}$ International Conference Engineering Mechanics 2017, Book of full texts: ISSN 1805-8248, ISBN 978-80-2014-54972, page 826-829, Svratka, Czech Republic, 15 (2017)

7. M. Romańczyk, Influence of gas inlet angle on the mixing process in a Venturi mixer, ITM Web of conferences Vol.15, 07005 (2017) - ISSN 2271-2097, DOI: 10.1051/itmconf/20171507005, CMES'17; Lublin, 23-25 November (2017)

8. S. Sundararaj, V. Selladurai, International Journal of Applied Engineering Research, 7, 891 (2008)

9. K. Ražniewić, Tablice cieplne z wykresami (Warszawa WNT 1966)

10. R. Absi, J. Appl. Mech., 75, 1 (2008). 